\title{
COVID-19 Data and the Cancer Patient: A Need for Registry Inclusion
}

\author{
Frederick Leslie Greene, MD, FACS (1) \\ Charlotte, NC
}

Disease is very old and nothing about it has changed. It is we who change as we learn what was formerly imperceptible.

- Charcot

The advent of hospital-based cancer registries initiated in the 1920s created data repositories that have been utilized for patient treatment, clinical research, and epidemiological study. As newer modes of treatment have been introduced, it has been critically necessary to develop coding structures that have allowed for appropriate inclusion into local, state, regional, national, and international cancer registries. The Centers for Disease Control and Prevention (CDC) spearheaded the development of cancer registries in every state, while the National Cancer Institute created the Surveillance, Epidemiology, and End Results (SEER) Program to enhance regional cancer reporting. ${ }^{1}$ In the late 1980s, the National Cancer Data Base (NCDB) was launched to serve as a repository for all cancer cases collected by hospitals accredited by the American College of Surgeons Commission on Cancer $(\mathrm{CoC}){ }^{2}$ Currently, there are approximately 39 million cancer cases in the NCDB.

While these data repositories were not extant during the flu pandemic of 1918-1919, the opportunity to collect relevant data from the COVID-19 pandemic is possible and critically important to pursue. As has been the case when new treatment methods, prognostic indicators, and cancer staging strategies have been initiated, the introduction of specific registry codes to allow for these COVID-19-related entries is necessary.

The ultimate work product for any of our local, state, and national registries begins with the ability of dedicated cancer registrars to enter cancer-specific information into

(C) Society of Surgical Oncology 2020

First Received: 21 April 2020;

Published Online: 28 May 2020

F. L. Greene, MD, FACS

e-mail: flgreene44@gmail.com abstracts that are based on software containing individual codes for data entry. In the US and Canada, these codes are periodically updated through the diligent work of standard setters working under the auspices of the North American Association of Central Cancer Registries (NAACCR) and the COC. Over the last few years, I have had the privilege to lead a work group with the goal of updating the COCproduced Facility Oncology Registry Data Standards (FORDS), which has now transitioned into the Standards for Oncology Registry Entry (STORE) and now serves as the coding structure for all COC-accredited hospital registries in the US. ${ }^{3}$

As we begin to recognize the ultimate consequences of infection with the SARS-CoV-2 virus on both operated and non-operated patients, it is mandatory to introduce a registry coding structure that can effectively collect data on all current and future patients. This work must be in concert with those who create electronic medical record technology and registry software. These codes must be aimed at identifying coronavirus-infected patients with a malignancy in order to capture data dealing with related comorbidities, TNM staging, recurrence, and overall survival, as well as treatment delays and other important sequelae occurring during this pandemic.

In my view, one of the barriers to allowing timely and meaningful cancer data collection has been the historical glacial approach to creating new coding structures for our hospital-based registries. We cannot afford to wait several years to see these codes brought to fruition. Critical losses of vital information relating to coronavirus infection affecting our cancer patients will be lost. We will lose valuable data that could be used for potential treatment strategies and epidemiological monitoring. The time for prospective inclusion of these data points is now, during the pandemic, not after vital information is lost. Our standard setters and organizations dedicated to cancer surveillance must act swiftly, along with other registry support groups, to create complete repositories that capture all relevant virus-associated data. We cannot afford to wait. 


\section{REFERENCES}

1. White MC, Babcock F, Hayes NS, et al. The history and use of cancer registry data by public health cancer control programs in the United States. Cancer. 2017;123(Suppl 24):4969-76.

2. Winchester DP, Stewart AK, Phillips JL, Ward EE. The National Cancer Data Base: past, present and future. Ann Surg Oncol. 2010;17:4-7.
3. Greene FL, Tedder P, Gress DM. Revision of the Facility Oncology data Standards (FORDS) Manual: a look to the future. J Registry Manag. 2014;4:209.

Publisher's Note Springer Nature remains neutral with regard to jurisdictional claims in published maps and institutional affiliations. 